\title{
Trente ans d'économie industrielle : réalisations et perspectives
}

Marco Bellandi, Sandrine Labory, Christian Longhi et Sylvie Rochhia

\section{(2) OpenEdition \\ 1 Journals}

Édition électronique

URL : https://journals.openedition.org/rei/4133

DOI : 10.4000/rei.4133

ISSN : 1773-0198

Éditeur

De Boeck Supérieur

Édition imprimée

Date de publication : 15 juin 2010

Pagination : 21-30

ISSN : 0154-3229

\section{Référence électronique}

Marco Bellandi, Sandrine Labory, Christian Longhi et Sylvie Rochhia, «Trente ans d'économie industrielle : réalisations et perspectives ", Revue d'économie industrielle [En ligne], 129-130 | 1er et 2e trimestres 2010, document 1, mis en ligne le 15 juin 2012, consulté le 02 juin 2022. URL : http:// journals.openedition.org/rei/4133; DOI : https://doi.org/10.4000/rei.4133 
Marco BELLANDI

Université de Florence

Sandrine LABORY

Université de Ferrara

Christian LONGHI, Sylvie ROCHHIA

Université de Nice Sophia Antipolis, GREDEG CNRS

\title{
TRENTE ANS D'ÉCONOMIE INDUSTRIELLE: RÉALISATIONS ET PERSPECTIVES
}

\begin{abstract}
P e numéro spécial a un caractère un peu particulier. Il ne traite pas d'un thème spécifique comme il est de tradition dans les numéros spéciaux de la Revue d'Économie Industrielle, mais il fait suite au colloque organisé pour ses trente ans. Dès lors son objet, trente ans d'économie industrielle, peut paraître ambitieux pour un simple numéro de revue. De fait, il ne s'agira pas de (re)faire un Traité d'Économie Industrielle, mais d'explorer quelques-unes des pistes essentielles qui se dégagent aujourd'hui. Le colloque a clairement mis en évidence la multiplicité des thèmes qui émergent des changements et défis auxquels sont confrontées les économies contemporaines et la multiplicité des tentatives de réponses. Un autre caractère particulier de ce numéro, lié à la nature du colloque auquel il est associé, tient à sa volonté d'analyser l'évolution de l'économie industrielle à travers ses publications dans les principales revues européennes qui lui sont consacrées, et d'évoquer les enjeux auxquels sont désormais confrontées ces revues.
\end{abstract}

Les divers changements - technologiques, organisationnels ou institutionnels - qui marquent nos économies peuvent avoir des échos tout aussi importants dans les théories que dans les revues. Les technologies de l'information et de la communication par exemple appellent à la définition de nouveaux modes de coordination, et par conséquent à un renouveau théorique de l'économie industrielle. Ces technologies transforment aussi les modes de diffusion des connaissances entraînant un changement des modes d'édition et des revues traditionnelles. La construction européenne, la régionalisation de l'économie mondiale, posent des questions au niveau des approches théoriques comme du fonctionnement des revues. Les problèmes de (re)localisation entraînés par l'évolution de l'Union européenne, questionnent les formalisations des dynamiques industrielles ainsi que la dimension nationale des revues. Ces « Trente ans d'économie industrielle » ont donc été l'occasion de faire un point sur les perspectives offertes tant au niveau des recherches qu'à celui de leur diffusion lors d'une table ronde des revues organisée pendant le colloque et présidée par Franco Malerba, éditeur de Industrial and Corporate Change. 
Les développements théoriques, politiques et empiriques en économie industrielle constituent le premier objet du numéro. Il s'agit de réfléchir aux nouveaux champs et questions de recherche que les changements des réalités industrielles et les avancées de cette discipline ont engendrés. Cette réflexion, que l'on découvrira dans la suite de ce numéro, est le fruit des contributions discutées lors du colloque. Avant de les présenter, cette courte introduction met en évidence comment l'évolution de la Revue s'inscrit dans l'histoire de l'économie industrielle et de ses développements.

La globalisation, la régionalisation, l'émergence d'une économie de la connaissance, le développement de technologies transversales, la crise enfin dans laquelle se sont enfoncées les économies motivent ces renouveaux. La crise surtout est souvent évoquée; mais comme l'a fait remarquer Franco Malerba lors de la table ronde, ce n'est pas tant la crise qui est importante, que les changements qui la provoquent et qu'elle entraîne en retour, changements qu'il s'agit de comprendre, et qui appellent souvent d'autres développements théoriques. Au sens large d'ailleurs, la naissance de l'économie industrielle moderne en France comme la création de la Revue d'Économie Industrielle ont constitué précisément un de ces développements. La Revue a largement contribué à la formation et à l'évolution de cette sous-discipline (Arena, 1999). Pour J. De Bandt (1991a), c'est bien la crise des années soixante-dix qui va imposer l'économie industrielle comme sous-discipline économique spécifique en France. Un de ses enjeux majeurs a résidé dans la définition de politiques industrielles idoines, à même de corriger ou d'adapter le fonctionnement du système productif. En effet, économie et politique industrielles sont quasiment jumelles, nées avec des objets et des objectifs communs, liés au souci de rendre compte avant tout du « monde réel » (Arena, 2006). « Les politiques industrielles ne devraient pas exister (...) dans un système économique réglé par les mécanismes de marché, organisé selon les lois de la concurrence, et dominé par le dogme de la propriété privée (...). Mais en fait dans un système économique de concurrence pure et parfaite, il n'y a pas place non plus pour l'économie industrielle » (Morvan, 1983 :19). Économie et politique industrielles ont de fait toujours renvoyé à des écarts au modèle idéal de la microéconomie, écarts qui sont la norme bien plus que l'exception. Les problèmes de crise, d'imperfection des marchés, de la concurrence... sont étroitement associés à la discipline, et les évolutions de la théorie se régulièrement traduites dans les politiques industrielles.

On ne fera pas dans ces quelques lignes d'introduction l'histoire des évolutions de la discipline, de la reformulation du paradigme «structures, comportements, performances » aux développements actuels. Arena (1999), le Traité d'Économie Industrielle (Arena et al., 1991a), mais surtout la série des numéros spéciaux de la Revue rendent compte de ces différents changements. La Revue a été à travers ses numéros spéciaux un révélateur de nouveaux 
domaines de recherche comme l'international (1980, 1991), les services (1988), la normalisation (1996), l'environnement (1998). Certains domaines, comme ceux de la politique industrielle $(1983,1995,2001)$ et de la politique de concurrence (1993) semblent plus traditionnels mais ont connu des changements réguliers. À travers ses numéros spéciaux, la Revue a aussi mis en évidence l'impact des nouvelles technologies sur la dynamique des marchés (2004), sur l'organisation de la production (1990) ou de certaines industries, comme la bio-industrie (1981), l'information et les communications (1987), les sciences du vivant (2007). Elle a enfin souligné le développement sans précédent des droits de propriété intellectuelle (2002) lié à l'essor des économies fondées sur la connaissance $(1999,2005)$. Ces numéros ont également recensé de nouvelles questions sur les plans analytique et méthodologique. Ainsi, la montée en puissance du rôle du changement technologique dans nos économies a contribué à favoriser les approches évolutionnistes dans les modèles d'analyse de l'innovation et de la production (1992). Quant aux questions de coordination entre agents, que ce soit au sein des firmes ou entre les firmes, elles ont été renouvelées par l'émergence d'une économie des contrats (2000) et des réseaux (2003), ainsi que par les modèles de jeux (2006).

Si la Revue s'est penchée sur de nombreux aspects de l'économie industrielle, elle a aussi cherché dès sa création à développer et à marquer certains de ses éléments clefs.

Le premier élément est à l'évidence l'approche en termes de méso-analyse. L'économie industrielle s'attache à expliquer les performances et le devenir du système productif, les activités industrielles et les services qui s'y rattachent au niveau des entreprises, des industries et des économies. Elle se réfère donc à la fois à des éléments ayant trait aux comportements des entreprises et à un ensemble de forces et de contraintes globales, que ni les approches micro-économiques ni les approches macro-économiques ne peuvent appréhender (De Bandt, 1991a). La réponse a été le développement d'une approche méso, précisée dès la naissance de la Revue par Yves Morvan (1977). Selon lui, il ne s'agit pas uniquement d'une analyse à un niveau intermédiaire, situé à mi-chemin entre micro et macro-analyse, mais d'une démarche qui s'efforce d'appréhender simultanément ces deux niveaux d'analyse. Le développement de cette méso-analyse se heurte pourtant à des difficultés et butte sur le manque de concepts adaptés, comme l'a souligné Morvan lui-même (1977). L'approche en termes de réseaux constitue de ce point de vue une voie possible pour dépasser ces limites. L'industrie n'a plus à être considérée comme un donné, son émergence et sa formation même peuvent être étudiées comme le "résultat progressif des interactions entre normes sociales et institutionnelles » (Cohendet et al., 2003 :30). Cet apport théorique, qui enrichit l'analyse de la dynamique et du changement, comme celle des politiques susceptibles d'être mises en œuvre, renouvelle les perspectives offertes à l'économie industrielle.

Le second est celui de la filière. Quatre rôles majeurs possibles lui ont été assignés: un outil de description technico-économique, une modalité de découpage 
du système productif, une méthode d'analyse de la stratégie des firmes, un instrument de politique industrielle (De Bandt, 1991b). En fait, les mêmes difficultés sur lesquelles a buté la méso-analyse ont quelque peu limité le développement du concept, souvent identifié à un champ extérieur à la sphère économique, celui des techniques de production (Arena et al., 1991b). L'ambition originale - définition d'un sous-système productif, comme ensemble organisé et finalisé de relations, doté d'une dynamique autonome, ouvert sur et en interaction avec d'autres sous-systèmes - pourrait trouver dans les approches en termes de réseaux des réponses pertinentes autorisant sa réalisation.

Les développements récents de l'activité économique appellent aussi un renouvellement de l'analyse en termes de filières et la considération de dimensions plus transversales. Suite à la combinaison des différentes bases scientifiques et techniques qui caractérise l'avancée des frontières de la connaissance dans les systèmes productifs et dans les produits, les frontières entre les secteurs sont dans une phase de porosité croissante. Les technologies de l'information, les nanotechnologies, les biotechnologies, les nouveaux matériaux irriguent plusieurs industries, filières..., très souvent en relation avec la $R \& D$ et les processus d'innovation qu'elles mettent en œuvre pour résoudre les interdépendances technologiques dans lesquelles elles s'insèrent. En d'autres termes, les secteurs ou les filières classiques deviennent interdépendants et ne sont plus des concepts pertinents lorsque les innovations technologiques sont transversales. Ces innovations sont des nœuds technologiques - des nœuds de réseaux - qui conduisent à reconsidérer les relations interentreprises et de sous-traitance.

Les tendances à l'innovation permanente et à l'internationalisation signifient enfin que la concurrence globale s'intensifie, que des relations nouvelles, que ce soit au niveau des entreprises, des institutions, des industries ou des localisations, se tissent. Les grandes entreprises doivent établir ou s'insérer dans des systèmes complexes leur associant des compétences externes et des implantations multiples; les localisations de l'industrie, surtout celles des petites entreprises, ont besoin de plates-formes pour les relations au niveau régional et international, entre des filières différentes, et avec la recherche et la formation.

Ces évolutions ont appelé de nouveaux modes de coordination. Un consensus existe depuis longtemps pour admettre que le marché ou les marchés constituent un mode de coordination des activités industrielles parmi d'autres. La filière a ainsi pu être considérée comme un ensemble structuré de modes de coordination par les prix de marché, par les conventions, par les contrats, par les règles et réglementations. En même temps que l'économie de marché s'étendait à l'ensemble des pays, le marché est de moins en moins apparu comme le mode de coordination privilégié des activités économiques. Le contrat a pris le pas dans cette coordination (Brousseau et Glachant, 2000). Cette « évidence empirique » a été mise en lumière par le développement de nouveaux courants, qui ont explicité ce mode de coordination et développé des cadres analytiques pouvant donner des représentations théoriques alternatives 
cohérentes du fonctionnement des économies de marché. Les approches contractuelles ont été à même de fournir les concepts permettant de mieux comprendre ces économies, et la construction d'une théorie économique des organisations fait désormais partie de l'agenda de l'économie industrielle. Depuis 30 ans, l'économie industrielle est donc passée d'une économie des structures à une économie des organisations et de la connaissance, caractérisée par l'importance des croyances collectives et des investissements immatériels, l'apparition de nouveaux marchés et de secteurs transversaux, le renouvellement des relations interentreprises et des stratégies de localisation .... Les agents, les entreprises sont reliés par des interactions sociales non contractuelles et décentralisées qui peuvent influencer de façon décisive le déroulement des transactions marchandes. La diversité de ces modes de coordination implique ainsi de reconsidérer les dynamiques d'innovation, de changement et de croissance.

Le troisième élément est la politique industrielle. Il faut attendre les années 1970 pour que la nécessité de coordonner et d'encourager la R\&D industrielle s'impose peu à peu notamment dans les secteurs de haute technologie. La logique sectorielle dans laquelle s'inscrit alors la politique industrielle est à relier avec la conception de l'économie industrielle qui prévaut dans ces années. Les filières constituent un ancrage pour ces politiques, qui vont toutefois évoluer dans les années 1980. La politique industrielle laisse progressivement la place à une politique de la concurrence, et cette évolution s'inscrit dans une conception renouvelée de la concurrence et des comportements. Bénéficiant des apports de la théorie des jeux, l'économie industrielle s'intéresse davantage aux stratégies d'acteurs, ce qui lui permet de mieux caractériser l'environnement concurrentiel et le fonctionnement des marchés. Dans les années 90, la logique strictement concurrentielle s'enrichit par la prise en compte des exigences de l'efficacité dynamique au niveau de la recherche et de l'innovation. Favoriser l'innovation et sa diffusion devient tout aussi important que promouvoir la concurrence. Les préoccupations de la dernière décennie se consacrent aux technologies dont la diffusion est horizontale de façon à toucher tous les secteurs. Les mécanismes interactifs ne relèvent plus de la seule logique concurrentielle et leur complexité s'accroît. Les avancées de la nouvelle microéconomie ou des évolutionnistes ne suffisent plus à comprendre ces phénomènes d'interaction aux multiples facettes.

Les « nouvelles » politiques industrielles font largement écho aux changements de l'organisation de l'industrie évoqués. Leur objectif est de concevoir les schémas incitatifs à même de créer des interactions entre les acteurs économiques et de développer les processus d'innovation et de création de connaissances. Ces politiques ne visent donc plus des entreprises ou des secteurs, à travers les champions nationaux ou les grands programmes traditionnels, mais des systèmes, le plus souvent localisés, associant une multiplicité d'acteurs et des compétences spécifiques. À titre d'exemple, les «pôles de compétitivité » en France, mais plus généralement les «stratégies de clusters » montrent bien comment les différents acteurs sont incités à former des liens et 
à construire des systèmes de production et d'innovation localisés, ouverts et solidement ancrés dans l'économie globalisée. La stratégie européenne en matière de politique industrielle, et la « méthode ouverte de coordination » sur laquelle elle s'appuie, illustrent cette problématique. Si elle reste basée sur la concurrence, elle passe également par la mise en réseau des institutions et des acteurs de tous les pays membres pour exploiter des synergies. La politique de la recherche et de l'innovation, la politique de promotion des PMI, la politique régionale et structurelle ont toutes pour objectif de réaliser des réseaux au niveau européen.

On attribue généralement cette nouvelle approche de la politique industrielle aux développements récents de l'économie géographique et aux travaux associés aux clusters Mais les développements de l'économie industrielle avaient abouti à des conclusions finalement très proches. Dès 1995, étudiant les processus de création de connaissances et de développement de compétences dans les entreprises, J. De Bandt (1995) met en évidence la nécessaire spécificité des politiques industrielles dans lesquelles on retrouve les logiques « bottom-up » ancrées dans les territoires, les environnements locaux et les systèmes incitatifs. Le territoire apparaît comme une construction, et les relations entre territoire et industrie, interdépendances et dynamiques, s'en trouvent profondément modifiées.

Les nouvelles politiques fusionnent les différentes dimensions des politiques, industrielle, technologique, régionale, dans une même politique dite industrielle. La définition, l'approfondissement et l'analyse de ces politiques, qui répondent aux évolutions des modes de coordination pertinents dans les économies contemporaines, font partie des questions fondamentales qui se posent aujourd'hui à l'économie industrielle.

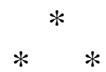

Les articles réunis dans ce numéro recouvrent chacun à leur manière plusieurs des perspectives analytiques identifiées ci-dessus. Cette partie dite « théorique » comporte douze contributions issues du colloque, toutes très riches; on ne pourra donc se permettre ici de rentrer dans le détail d'aucune d'entre elles, auxquelles on renvoie bien évidemment le lecteur. On se contentera d'expliciter la logique du sommaire proposé et leur enchaînement.

Que le premier article de ce numéro soit une contribution de George B. Richardson s'imposait. George B. Richardson a en effet marqué la recherche à double titre: par ses travaux sur la firme et la coordination et par son rôle au niveau de la valorisation de la recherche en tant que directeur général d'Oxford University Press. Sa contribution reflète cette double implication dans l'économie industrielle.

Les autres contributions sont ensuite organisées selon les thèmes suivants. 
La première partie se consacre à l'étude des différents modes de coordination et aborde des questions longtemps en débat ou des problèmes nouveaux que les évolutions récentes ont soulevés. Jacques Cremer revient sur la question de l'intégration verticale et pose de manière originale le problème de l'intervention sélective. Benjamin Coriat et Olivier Weinstein s'interrogent sur les avancées récentes en matière de théories de la firme. Ces deux premières contributions éclairent l'actualité des recherches fondamentales relatives aux approches de la firme. Les deux suivantes se penchent sur les questions relatives au marché. Éric Brousseau et Antonio Nicita mettent en évidence les apports de la Nouvelle Économie Institutionnelle au niveau de l'organisation et de l'efficience du marché. Étudiant la régulation des nouveaux marchés du numérique, Philippe Barbet et Isabelle Liotard soulignent l'importance des questions liées à la propriété intellectuelle.

La deuxième partie examine la question des réseaux à travers la mise en évidence de quelques problématiques importantes. La question du rôle que peuvent jouer les communautés, les différents groupes sociaux dans les processus de création de connaissances est clairement posée par Patrick Cohendet, Patrick Llerena et Laurent Simon. Michel Ferrary montre que l'utilisation de la sociologie économique dans ce type d'étude peut se révéler fondamentale. Enfin, les réseaux sont au cœur des développements récents de la théorie des territoires et du renouveau de géographie économique de l'innovation comme le démontrent Corinne Autant-Bernard, Pascal Billand et Nadine Massard.

La troisième partie est consacrée aux politiques industrielles. Nick von Tunzelman soulève la question du fondement théorique de la politique industrielle et soutient que les « défaillances de réseaux » sont tout aussi importantes que les « défaillances de marché » dans la justification théorique des politiques publiques. Marco Bellandi et Annalisa Caloffi mettent en avant les défis auxquels sont aujourd'hui confrontés les districts industriels italiens et examinent les différentes possibilités d'action au niveau des politiques publiques. Frédéric Marty et Julien Pillot montrent que dans les affaires de concurrence, l'application du concept de facilités essentielles est loin d'être évidente et peut générer des comportements opportunistes comme l'atteste le cas Microsoft. Enfin, Patrizio Bianchi et Sandrine Labory soulignent l'intérêt d'une approche classique considérant la politique industrielle comme un programme global de développement industriel et économique.

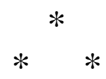

À l'instar de tous les éditeurs scientifiques, les revues d'économie industrielle ont toujours placé les avancées de la recherche au centre de leur chaîne éditoriale. C'est notamment ce que l'on constate en retraçant l'évolution des publications dans chacune d'entre elles. Les contributions qui constituent le second volet de ce numéro s'inscrivent dans cette perspective. 
Ce second volet s'ouvre sur une contribution de Jacques De Bandt qui a dirigé la Revue d'Économie Industrielle pendant plus de 30 ans et qui fait un état des avancées et des échecs de l'économie industrielle d'aujourd'hui.

Patrizio Bianchi, Sandrine Labory et Enzo Pontarollo proposent une analyse de la politique industrielle en Italie au travers des articles que l'Industria a consacrés à ce thème depuis 30 ans. Michel Rainelli s'intéresse à l'intérêt que la Revue d'Économie Industrielle a accordé au droit économique et à la politique de la concurrence. Richard Arena et Cindy Navarro étudient les articles publiés par la Revue d'Économie Industrielle depuis 1977 et mettent en évidence les thèmes et corpus théoriques qui ont émergé ou se sont effacés au cours du temps.

L'examen des publications dans les revues montre ainsi que leur histoire reste marquée par l'apparition de nouveaux problèmes mais surtout peut-être par l'apparition dans leur champ éditorial de nouveaux objets que l'on croyait réservés à d'autres disciplines. De fait le paysage qu'offre aujourd'hui l'économie industrielle est plus hétérogène que jamais.

En écho à la contribution de Jacques De Bandt, Francesco Malerba a souligné, lors de la table ronde qu'il a présidée, que la discipline a d'une certaine façon « échoué » car elle n'a pas réussi à fédérer la communauté des chercheurs sur des représentations plus pertinentes de la réalité industrielle. L'économie industrielle se serait peu à peu éloignée de ce qui avait fondé son originalité, à savoir une approche " empirically driven », dans laquelle ce qui importe, ce n'est pas la pertinence empirique des implications de ses modèles mais plutôt celle de ses hypothèses. Pour Francesco Malerba, Industrial and Corporate Change veille à suivre cette politique éditoriale. Dans ce champ régulièrement renouvelé, il propose de définir l'économie industrielle comme une discipline axée sur l'étude du changement (au niveau des firmes, de l'industrie, ...) et caractérisée par l'interdisciplinarité (histoire, économie, management...) et l'ouverture théorique. Une telle approche de l'économie industrielle serait incontournable lorsqu'il s'agit de couvrir les questionnements actuels.

Enfin, la table ronde a été l'occasion de discuter du devenir des revues et des différents enjeux de l'édition scientifique. Suite au développement des TIC et à l'apparition de nouveaux modes de valorisation de la recherche comme les archives ouvertes, les éditeurs ont été confrontés à l'évolution de leur modèle économique. Au niveau de l'organisation du processus éditorial, les TIC ont permis de faciliter la gestion des abonnements ou encore la conservation des archives, de simplifier le travail des comités de lecture sans pour autant transformer la chaîne éditoriale qui reste articulée autour de ces comités. Les comités constituent à la fois des ressources et des contraintes pour l'activité scientifique et sont au cœur du processus de validation des savoirs. Les revues qui organisent ce processus de validation apparaissent donc comme un des acteurs essentiels de la chaîne éditoriale. 
Mais en publiant les résultats de différents travaux de recherche qu'elles certifient et valorisent, les revues ne s'adressent pas seulement à la communauté des scientifiques. Comme le rappellent les éditeurs, une revue cherche aussi à contribuer au débat économique en ajoutant aux articles scientifiques des chroniques davantage ancrées sur l'actualité, ou des contributions visant à répondre à tel ou tel point de la demande sociale. Or, il ressort de cette discussion que la plupart des revues non anglophones rencontrent des difficultés à prendre une part active dans les débats internationaux en terme de visibilité des articles qu'elles publient. À ces difficultés s'ajoutent les changements qui affectent le monde de l'édition, notamment les transformations éditoriales induites par les supports numériques.

Les prix pratiqués par les grands groupes éditoriaux et le développement des offres numériques ont en effet poussé les bibliothèques à se tourner vers les bouquets de revues ou les agences d'abonnement. Les universités, en tant que producteurs et diffuseurs de connaissances, jouent un rôle clef dans ce mouvement. Il en est ainsi de Revel, le portail de revues en sciences humaines et sociales de l'université de Nice-Sophia Antipolis, créé pour développer un outil de publication électronique dans l'esprit des archives institutionnelles ouvertes. Sans entrer dans une discussion sur le mouvement de l'accès ouvert, les revues d'économie industrielle ne peuvent ignorer certains de ces enjeux, notamment ceux qui touchent au fonctionnement traditionnel des revues et aux modalités de leur évaluation. Les revues présentes à la table ronde ont recours à la procédure basée sur l'expertise par les pairs (peer-reviewing) avant publication. Le développement des archives ouvertes inverse le processus d'édition qui démarre désormais par la publication du texte indépendamment d'une validation organisée. Ce changement interroge les revues sur l'évolution de leurs procédures classiques éditoriales. Premièrement, les règles de l'anonymat sont de plus en plus difficiles à respecter. Deuxièmement, l'évaluation par les pairs n'est plus simplement effectuée au sein d'un comité d'experts mais tend à s'étendre au niveau de la communauté des lecteurs; par conséquent, le recours à des modèles de validation ex post (après publication) comme ceux basés sur la mesure de l'impact d'un article tend à se répandre. Si ces modèles de validation ex post venaient à se généraliser, la diffusion de l'article deviendrait un élément important de son éventuel classement.

Compte tenu de ces enjeux en termes de diffusion, les quatre revues non anglophones ont souhaité coopérer pour donner une visibilité accrue aux analyses et débats qu'elles diffusent dans leur langue nationale et développer de nouvelles procédures éditoriales.

L'idée d'un réseau européen de revues d'économie et de politique industrielles est apparue suite à une série d'échanges entre les membres d'Economia e politica industriale, de l'Industria, d'Economia Industrial, et de la Revue d'Économie Industrielle. L'objectif est d'intervenir conjointement sur des thèmes à visée européenne pour montrer comment chacune des revues appréhende les problèmes actuels des entreprises et de l'industrie et quelles sont les solutions proposées dans chacun des contextes économiques. Une attention 
particulière est ainsi accordée aux conséquences des politiques économiques régionales, nationales et territoriales - sur la compétitivité des systèmes productifs, le développement de nouvelles industries, et la prospérité des territoires qui les supportent. Le réseau constitué se propose de conjuguer analyse théorique et analyse comparative des divers aspects des économies espagnole, française et italienne pour offrir aux entrepreneurs et aux responsables de politique économique des réflexions et suggestions de politiques utiles et directement applicables. Ce nouveau mode de diffusion du savoir s'appuiera sur une publication électronique dont la mission ne sera pas de numériser des informations diffusées au sein des revues existantes mais de créer un espace collaboratif au sein du réseau. Comme le souligne Berthou (2009), la publication peut alors dépasser le dispositif de la revue traditionnelle et mobiliser d'autres formes éditoriales que celles de l'article et devenir un instrument de mise en réseau de la recherche. L'objectif de cette publication électronique serait ainsi de constituer une communauté virtuelle pour favoriser les échanges et la mise en commun des résultats de la recherche en économie industrielle.

\section{BIBLIOGRAPHIE}

ARENA R. (1999), «Un changement dans l'orientation de la Revue d'Économie Industrielle », Revue d'Économie Industrielle, $\mathrm{n}^{\circ} .87$, pp. 7-30.

ARENA R. (2006), « Avant-propos: pour une étude renouvelée des comportements en économie industrielle » Revue d'Économie Industrielle, $\mathrm{n}^{\circ}$ 114-115 (2-3), pp. 15-20.

ARENA R., BENZONI L., De BANDT J., ROMANI P.M. (eds) (1991a), Traité d'économie

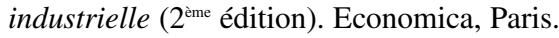

ARENA R., RAINELLI M., TORRE A. (1991b), « Filières et découpages productifs, in Traité d'économie industrielle » (2 ${ }^{\text {ème }}$ édition), R. Arena, L. Benzoni, J. De Bandt et P.M. Romani (eds). Economica, Paris, pp. 239-250.

BERTHOU B. (2009), « La publication numérique en sciences humaines et sociales: vers un nouveau modèle de diffusion du savoir? », Mémoires du livre/Studies in Book Culture, vol. 1, $\mathrm{n}^{\circ}$ 1. http://www.erudit.org/revue/memoires/2009/v1/n1/index.html

BROUSSEAU E., GLACHANT J.M. (2000), «Économie des contrats et renouvellements de l'analyse économique », Revue d'Économie Industrielle, $\mathrm{n}^{\circ} 92$ (2-3), pp. 23-50.

COHENDET P., KIRMAN, A., ZIMMERMANN, J.-B. (2003), « Émergence, formation et dynamique des réseaux - Modèles de la morphogenèse », Revue d'Économie Industrielle, $\mathrm{n}^{\circ} 103(2-3)$, pp. 15-42.

De BANDT J. (1991a), «L'économie industrielle dans le contexte français: développements et spécificités », in Traité d'économie industrielle ( $2^{\text {ème }}$ édition), R. Arena, L. Benzoni, J. De Bandt et P.M. Romani (Eds). Economica, Paris, pp. 156-170.

De BANDT J. (1991b), «L'économie industrielle face à la réalité des transformations industrielles », in Traité d'économie industrielle (2 $2^{\mathrm{eme}}$ édition), R.Arena, L. Benzoni, J. De Bandt et P.M. Romani (eds). Economica, Paris, pp. 867-934.

De BANDT J. (1995), «Quelles conceptions des entreprises, des systèmes et des processus productifs inspirent quelles politiques industrielles? », Revue d'Économie Industrielle, $\mathrm{n}^{\circ} 71$ 1er trimestre, pp. 123-141.

MORVAN Y. (1977), «À propos de l'économie industrielle », Revue d'Économie Industrielle, $\mathrm{n}^{\circ} 1$ (1), pp. 5-26.

MORVAN Y. (1985), Fondements d'économie industrielle, Paris, Economica, collection Gestion. 